\title{
Advances in Fire Debris Analysis
}

\author{
Michael E. Sigman ${ }^{1,2, *}$ and Mary R. Williams ${ }^{1}$ \\ 1 National Center for Forensic Science, University of Central Florida, P.O. Box 162367, \\ Orlando, FL 32816-2367, USA; mary.williams@ucf.edu \\ 2 Department of Chemistry, 4111 Libra Drive, Physical Sciences Bld. Rm. 255, Orlando, FL 32816-2366, USA \\ * Correspondence: michael.sigman@ucf.edu; Tel.: +1-407-823-6469
}

Received: 2 January 2019; Accepted: 21 February 2019; Published: 26 February 2019

The practice of forensic fire debris analysis and data interpretation in operational (i.e., casework) laboratories has gone without significant change since the widespread adoption of gas chromatography-mass spectrometry (GC-MS) and a consensus standard for interpretation from the American Society for Testing and Materials (ASTM). The industry standard for fire debris analysis is ASTM E1618, "Standard Test Method for Ignitable Liquid Residues in Extracts from Fire Debris Samples by Gas Chromatography-Mass Spectrometry" (GC-MS) [1]. The first version of the ASTM E1618 was approved in 1997. Since then, there has been some minimal reshuffling of the ignitable liquid classes, with a few class additions and deletions, but no significant changes otherwise. The ASTM standard defines classes of ignitable liquids based on several criteria, including product, process, chemistry, etc. Only one ASTM E1618 ignitable liquid class is currently product-based (gasoline), while one is process-based (petroleum distillates), four are chemistry-based (normal alkane, isoparaffinic, naphthenic paraffinic and aromatic products), and one exhibits no class characteristics or characteristics of multiple classes (others-miscellaneous). An additional class, the oxygenated solvents class, may possess the characteristics of any of the other classes, or multiple classes, so long as the solvent contains a major contribution from an oxygenated compound (alcohol, ketone, ether, ester, etc.). However, gasoline samples containing a significant ethanol component (gasohol) are classified as gasoline, not oxygenated solvents. The classification scheme can be confusing, yet the class of a liquid may be helpful to investigators wishing to identify commercial products.

Under the ASTM E1618, target compounds are only designated for the gasoline class, as well as the medium and heavy subclasses of petroleum distillates class (i.e., those in the $\mathrm{C}_{8}-\mathrm{C}_{20+}$ range). The standard provides for the use of "target compound chromatograms" [2]; however, these are rarely used by analysts. The ASTM standard directs the analyst to visually recognize the presence of class-associated chromatographic patterns in fire debris GC-MS total ion chromatograms and extracted ion profiles as a means of determining the presence of ignitable liquid residues. Complicating this procedure is the persistent and highly diverse background arising from the pyrolysis (thermal breakdown) of building materials and furnishings, the weathering of ignitable liquids (evaporation) and potential biological degradation of ignitable liquid residues in the sample. The laboratory weathering of ignitable liquids for comparison purposes is time and labor intensive, and difficult to accurately reproduce.

The forensic analyst must work through a complicated data set and arrive at a categorical decision and statement (report) regarding the presence or absence of an ignitable liquid. Decisions and statements are softened only by qualifying statements that can be biasing (i.e., "[T]the absence of an ignitable liquid residue does not preclude the possibility that ignitable liquids were present at the fire scene. Ignitable liquids are volatile compounds that may have evaporated, been totally consumed in a fire, environmentally altered or removed, or otherwise indistinguishable from background materials" [1]). Current methods in forensic fire debris data interpretation are without validation, 
and yet the resulting reports and testimony are accepted in court proceedings without an associated error rate or statement of evidentiary value.

This special edition of Separations presents current research efforts that touch on some of the challenges mentioned in the introductory paragraphs. We hope that the work presented in this special issue will inspire young researchers to accept the challenge of tackling difficult problems in forensic science, where their work can make a difference in the lives of those who have been victimized or falsely accused.

Target compounds are highly useful in the determination of gasoline residues in fire debris, but somewhat less useful for other ignitable liquid classes. Identifying the presence of target compounds that are often found in ignitable liquids, but rarely observed in substrate pyrolysis, could assist in the identification of ignitable liquid residues in fire debris. However, the utility of target compounds can be limited in one-dimensional chromatography, where their identification may be complicated by resolution and peak capacity limitations. This is especially true in isoparaffinic, naphthenic paraffinic and distillate classes where peaks are often poorly resolved. Sampat and coworkers address this issue through the application of comprehensive GC $\times$ GC-MS to the analysis of gasoline, white spirit and lamp oils in the presence of pyrolysate [3]. The increased peak capacity in comprehensive chromatography facilitates the identification of selected analytes in fire debris, resulting in an improved capability to detect ignitable liquid residues. Research reported in this special issue by Nizio and coworkers further explored the enhanced analytical characteristics offered by GC $\times$ GC-MS for the detection of ignitable liquid residues from burned bodies [4]. Headspace samples were collected from body bags containing burned porcine corpses and analyzed for the presence of ignitable liquid residue. The headspace samples were thermally desorbed and analyzed by comprehensive gas chromatography with a time-of-flight mass spectrometer for detection. Gasoline residues were detected from bodies burned with the aid of the liquid, and not detected from samples burned without the aid of the liquid or from empty body bag controls. Comprehensive gas chromatography, coupled with time-of-flight mass spectrometry, was shown by Abel and coworkers to provide lower limits of identification by an order of magnitude, relative to GC-MS identification performed by trained analysts for neat $75 \%$ evaporated gasoline and $25 \%$ evaporated diesel samples [5]. The limits of identification for gasoline were determined to be approximately $6.2 \mathrm{pL}$ on-column for both the neat gasoline and samples containing pyrolysate interferences. These three papers demonstrate the potential for advances in fire debris analysis by incorporating technology that is commercially available today.

The heat from a fire often results in the partial evaporation of any ignitable liquid present during the fire. The evaporation of an ignitable liquid results in the reduction of the more volatile components, which will correspond with an enhancement in the relative contribution of the less volatile components. The resulting chromatographic distortion complicates the process of identifying an ignitable liquid based on chromatographic patterns. Furthermore, evaporation complicates the preparation of an exemplar for reporting or testimony purposes. The standard, ASTM E1618, requires that a reference ignitable liquid be analyzed under similar conditions as the casework sample for comparison and classification purposes. When the casework sample is partially evaporated, it becomes necessary to prepare a comparable reference sample. McIlroy and coworkers address the need to prepare accurately evaporated samples by numerically estimating the chromatographic profile of a partially evaporated ignitable liquid for comparison with the residue contribution of a casework sample [6].

Reporting and testimony under current practices require the analyst to make a categorical decision regarding the presence or absence of ignitable liquid residues. When reporting samples that are determined to be positive for ignitable liquid residues, the standard stipulates that there is to be no implied difference in the perceived level of confidence [1]. Current trends in forensic science and legal practice in Europe are moving toward the use of probabilistic statements that reflect evidentiary value [7]. While the U.S. appears to be moving at a slower pace with regard to probabilistic statements, the need for more method validation and statistical data interpretation has been recognized and widely reported by the National Academies of Science [8]. Allen and coworkers address the issue of calculating 
likelihood ratios for fire debris evidence, probabilistic reporting and the influence of ignitable liquid population distributions on the accuracy of methods [9]. The likelihood ratio work addresses the challenge of chromatographic retention time shifts by basing the chemometric calculations on a mass spectrum averaged across the chromatographic profile. In related work reported in this special edition, Aliaño-González and coworkers report on the use of headspace-gas chromatography-ion mobility spectrometry (HS-GC-IMS), coupled with hierarchical clustering and linear discriminant analysis, to discriminate between samples that were burned with and without ignitable liquid [10]. The HS-GC-IMS method was also able to discriminate between four different ignitable liquids (gasoline, diesel, ethanol and paraffin). In other machine learning research, Thurn and coworkers report the use of unsupervised learning by Kohonen self-organizing maps, coupled with selected ion intensities from GC-MS data, to organize neat ignitable liquids into their ASTM E1618 classes and to separate those IL classes from pyrolyzed substrate samples [11]. These contributions to this special issue are representative of a growing body of research that makes use of computational and chemometric-aided data analysis to assist in the interpretation of fire debris data.

Acknowledgments: The editorial team would like to thank all of authors for their excellent contributions to this special issue. The efforts of the reviewers are also acknowledged as contributing greatly to the quality of this special issue. Finally, M.E.S. and M.R.W. acknowledge and thank the staff at Separations/MDPI for their assistance.

Conflicts of Interest: The authors declare no conflict of interest.

\section{References}

1. ASTM E1618-14 Standard Test Method for Ignitable Liquid Residues in Extracts from Fire Debris Samples by Gas Chromatography-Mass Spectrometry; ASTM International: West Conshohocken, PA, USA, 2014.

2. Lennard, C.J.; Tristan Rochaix, V.; Margot, P.; Huber, K. A GC-MS database of target compound chromatograms for the identification of arson accelerants. Sci. Justice 1995, 35, 19-30. [CrossRef]

3. Sampat, A.; van Daelen, B.; Lopatka, M.; Mol, H.; van der Weg, G.; Vivó-Truyols, G.; Sjerps, M.; Schoenmakers, P.; van Asten, A. Detection and characterization of ignitable liquid residues in forensic fire debris samples by comprehensive two-dimensional gas chromatography. Separations 2018, 5, 43. [CrossRef]

4. Nizio, K.; Forbes, S. Developing a method for the collection and analysis of burnt remains for the detection and identification of ignitable liquid residues using body bags, dynamic headspace sampling, and TD-GC $\times$ GC-TOFMS. Separations 2018, 5, 46. [CrossRef]

5. Abel, R.; Zadora, G.; Sandercock, P.; Harynuk, J. Modern instrumental limits of identification of ignitable liquids in forensic fire debris analysis. Separations 2018, 5, 58. [CrossRef]

6. McIlroy, J.; Smith, R.; McGuffin, V. Fixed- and variable-temperature kinetic models to predict evaporation of petroleum distillates for fire debris applications. Separations 2018, 5, 47. [CrossRef]

7. Champod, C.; Biedermann, A.; Vuille, J.; Willis, S.; De Kinder, J. ENFSI guideline for evaluative reporting in forensic science, a primer for legal practitioners. Crim. Law Justice Wkly. 2016, 180, 189-193.

8. Council, N.R. Strengthening Forensic Science in the United States: A Path Forward; The National Academies Press: Washington, DC, USA, 2009; p. 348.

9. Allen, A.; Williams, M.; Thurn, N.; Sigman, M. Model distribution effects on likelihood ratios in fire debris analysis. Separations 2018, 5, 44. [CrossRef]

10. Aliaño-González, M.; Ferreiro-González, M.; Barbero, G.; Palma, M.; Barroso, C. Application of headspace gas chromatography-ion mobility spectrometry for the determination of ignitable liquids from fire debris. Separations 2018, 5, 41. [CrossRef]

11. Thurn, N.; Williams, M.; Sigman, M. Application of self-organizing maps to the analysis of ignitable liquid and substrate pyrolysis samples. Separations 2018, 5, 52. [CrossRef]

(C) 2019 by the authors. Licensee MDPI, Basel, Switzerland. This article is an open access article distributed under the terms and conditions of the Creative Commons Attribution (CC BY) license (http://creativecommons.org/licenses/by/4.0/). 